\title{
Bericht über die Sitzung der Moskauer augenärztlichen Gesellschaft vom 28. April (11. Mai) 1909
}

Vor der Tagesordnung finden 2 Krankendemonstrationen statt:

Dr. W. Strachow stellte eine 45 jährige Frau mit dem Bilde der beider-seitigen Papillitis vor. Sehschärfe und Gesichtsfeld normal. Nervensystem, Ohren, Nase, Nieren ohne Befund. Frühzeitiges (mit 38 Jahren) Sistieren der Menses. Die Kranke steht schon 1 Jahr unter der Beobachtung und bleibt der Augenbefund während dieser Zeit immer derselbe. Hg- und KJ-Kur haben keinen Einfluss geübt. Trotz der normalen Sehschärfe und des normalen Gesichtsfeldes spricht sich Strachow vorsichtig bezüglich der Prognose aus und erinnert an den in derselben Gesellschaft vorgestellten Fall von Natanson jun., wo die Sehschärfe ebenso lange normal blieb und wo es doch später zur Sehnervenatrophie kam.

Dr. V. Odinzow demonstriert einen 9 monatlichen Albino. Haare voll-kommen pigmentlos. Iris hellblau. Augenhintergrund typisch albinotisch. Nystagmus horizontalis. Familienanamnese belanglos. Der ältere Bruder normal.

Dr. A. Natanson sen. Kurze Bemerkung, eine Kranke mit beider-seitigem pulsierendem Exophthalmus betreffend. Die Kranke wurde im Jahre 1908 in der Sitzung vom 29. IV. demonstriert. Schon damals sprach der Vortragende die Vermutung aus, dass es sich um ein Aneurysma der Carotis interna im Sinus cavernosus infolge eines Trauma handelt. Im Januar 1909 erschien die Kranke wieder mit grossen pulsierenden Erweite-rungen der Venae ophthalm. superiores im oberen inner en Winkel beider Orbitae. Bei der Auskultation systolisches Geräusch, links starker wie rechts. Cyanose im Gebiete der Vena facial, anter., die bekanntlich mit der V. ophth. super, anastomosiert. Alles das stellt die Diagnose des Aneurysma arteriovenosum ausser Zweifel. Auf die Operation ging die Kranke nicht ein.

Dr. A. Natanson sen. berichtet ferner über 1 Fall von Keratitis paren-chymatosa im Anschluss an ein Trauma und bespricht die Möglichkeit des Zusammenhanges zwischen dem letzteren und der Entwicklung der paren-chymatösen Hornhautentzündung.

In dem vorgetragenen Falle entwickelte sich die Keratitis bei einem Lokomotivführergehilfen einen Monat, nachdem ihm in das linke Auge ein Kohlenstück geflogen. Symptome der Lues hereditaria waren vor-handen. Rasche Besserung auf die spezifische Behandlung. Vortragender kommt zu dem Schlusse, dass ein Trauma einen Anstoss zur Entwicklung der Keratitis bei den dazu disponierten Individuen abgeben kann, und weist auf die Wichtigkeit dieses Umstandes bei der Beurteilung forensischer Fälle hin.

In der darauf folgenden Diskussion gingen die Meinungen auseinander. Die einen (Loschetschnikoiv, Lawrentjew, Maklakow u. A.) stimmten dem Vortragenden zu und führten aus ihren Beobachtungen ähnliche Fälle an, die anderen lehnten aber entweder jeden Zusammenhang ab (Awerbach, Fëdorow, Strachow) oder sprachen sich sehr zurückhaltend aus (Gurwitsch, Jachontow), indem sie auf die in Fallen dieser Art nötige Vorsicht und auf das Bestreben der Kranken, jede Augenerkrankung in Zusammenhang mit einer Verletzung zu bringen, hinweisen. 
Dr. Natanson sen. demonstriert mikroskopische Präparate eines Falles von Lipodermoiden der beiden Bindehäute, die bei einem 60 jährigen Manne, welcher ausserdem an Ohrsklerose und Lipom im Skapulargebiete leidet, entfernt wurden. Die Geschwülste befanden sich unter der Bindehaut aussen oben und waren mit der letzteren nicht verwachsen. Mikroskopisch: Fett-, Muskel- und Nervengewebe und acino-tubulöse Drüsen.

Dr. Awerbach berichtet über einen Fall von lymphatischen Cysten der beiden Konjunktivae. Bei dem 59 jährigen Kranken, der an Trachom leidet, waren die beiden unteren Uebergangsf alten von symmetrischen Geschwülsten eingenommen. Die Geschwülste waren wurstförmig, lagen unter der frei beweglichen Bindehaut und liessen sich leicht herauspräparieren. Mikroskopisch eine Cyste, deren Wand mit zylindrischen Zellen ähnlich dem Endothel der Lymphgefässe bekleidet war. Die Zellen sassen auf einer Membrana basilaris und gingen an einer Stelle in das Endothel eines Lymph-

Zeitsohrift für Augenheilkimde. Bd. XXII. Heft 1.

6

82

Unfall- und Versicherungskunde.

gefässes über. Vortragender betrachtet seinen Fall als zu dem Typus deГ lymphatischen Cysten nach Gírincíone gehörend. In der Literatur konnte er nur 13 Fälle dies $\beta r$ Art ermitteln.

Lokalisation in den unteren Uebergangs-falten gewöhnlich zwischen der Cornea und Caruncula) und die grosse Aus-dehnung zeichnen seinen Fall von den friiher beschriebenen aus.

Dr. Smolianinow $\cdot$. Ein Fall von traumatischer Neurose. Bei einem Eisen-bahnbediensteten entwickelten sich 11/2 Monate nach einer Kopfquetschung die Erscheinungen von hysterischer Hemichorea rechts. Objektiv ohne Befund. Von Seiten der Augen war zu bemerken, dass das Gesichtsfeld für Grün weiter als für Rot war. Sonst keine Abweichungen. Sehschärfe und Gesichtsfeld für Weiss normal.

Dr. Odínzow demonstriert mikroskopische Präparate einer epithelialen Cyste in der Vorderkammer. Das Auge wurde wegen Sekundärglaukoms 7 Jahre nach einer Verletzung enukleiert. Das Epithel kleidete die Vorderkammer beinahe in ganzer Ausdehnung aus und liess nur ihren äusseren oberen Quadranten frei, der von der übrigen Rammer durch zwei vordere Synechien getrennt war. Das Epithel erinnerte sehr an das derKonjunktiva. Spärliche Becherzellen. Stellenweise hydropische Degeneration. Die Ent-wicklung dieser Cysten wird vom Vortragenden im Sinne Stöltings erklärt, nämlich durch unmittelbare Einwucherung des Epithels den Wundkanal entlang bis zu der der inner en Wundöffnung anliegenden Iris. Ob in seinem Falle das Glaukom durch Epithelwucherung im Kammerwinkel hervor-gerufen war, lässt Vortragender unentschieden, da ausserdem Iriseinklem-mung und vordere Synechien vorhanden waren.

Dr. A. Natanson sen.: Entfernung eines Eisenstückes aus der Regen-bogenhaut, (mit Demonstration des Fremdkörpers und mikroskopischerPräparate des exzidierten Irisstückes mit deutlicher Eisenreaktion). DerFremdkörper war fest in die Iris eingekeilt und wurde mittels Iridektomieentfernt. Bei der Untersuchung mit dem Volkmannsche $\tau$ i Elektromagnetenmachte die Iris eine deutliche Bewegung nach vorn, aber es trat keineSchmerzempfindung auf. Vortragender weist auf die Unzuverlässigkeitder Schmerzreaktion hin und spricht sich zu Gunsten des Sideroskops undder Röntgenaufnahme bei der Diagnosestellung von Fremdkörpern imAuge aus. Dr. V. Odínzow.

Unfall- und Versicherungskunde. 
G. W. Walibaum, Nervenarzt, Sanatorium Waldpark-Schierke: Zur Frage der Tabes traumatiea. Ärztliche Sachverständigen-Zeitung. 1909. No. 9.

Verf. erörtert die Frage im Anschluss an einen von ihm beob-achteten Fall:

Ein 46 jähriger Lokomotivführer, der niemals an Syphilis gelitten, niemals Missbrauch mit Alkohol getrieben haben will, öfters an Kopfschmerz und Schlaflosigkeit, aber nie an ernsten nervösen Be-schwerden litt, war bei zwei Unfällen beteiligt:

Am 10. IV. 1904 fiel er von seiner Maschine und schlug mit dem Rücken gegen eine eiserne Barriere. Er war 14 Tage krank wegen Kreuz-schmerzen und Rückenquetschung, konnte dann aber wieder ohne Be-schwerden Dienst tun.

A.1. Am 1. VI. 1904 hatte er einen zweiten Unfall: Zwei Maschinen fuhren auf die seinige auf. Er wurde auf seiner Lokomotive hin- und her-geschleudert

ohne äusseren Schaden zu nehmen; er wurde jed'och durch den Unfall seelisch aufs äusserste erschüttert.

Es bestand unmittelbar nach dem Unfall: heftiges Zittern der Beine

Kopf- und Kreuzschmerzen. Kein objektiver Befund am Nervensystem. 